\title{
Modellbildung und Simulation als Grundlagenfach
}

\author{
Werner Maurer \\ Zürcher Hochschule für Angewandte Wissenschaften (ZHAW); werner@pegaswiss.ch
}

\begin{abstract}
Zusammenfassung. Modellbildung und Simulation öffnen neue Türen, sobald der explorative Charakter dieser Methode erkannt und zum Kompetenzerwerb eingesetzt wird. Unsere Erfahrungen aus den letzten dreissig Jahren zeigen, wie sich die studentischen Aktivitäten vom Lösen von Standardaufgaben hin zur kreativen Auseinandersetzung mit den Grundgesetzen verschieben, wenn man die entsprechenden Aufgaben stellt und die Ergebnisse mündlich und schriftlich einfordert. Zur Hydrodynamik, Translationsmechanik und Thermodynamik wird nachfolgend je eine kurze Einführung in die Theorie gegeben und anhand einer Aufgabenstellung erläutert. Wie man all diese Strukturen in eine Modelica-Bibliothek einbringt und so für das weitere Studium nutzbar macht, wird mangels Erfahrung nur als Idee skizziert.
\end{abstract}

\section{Einführung}

Viele Studiengänge speziell an Fachhochschulen sind stark fragmentiert, was die Stoffmenge aufbläht und nach jedem Semester zu einem Prüfungsmarathon führt. In Folge der fortschreitenden Spezialisierung und unter dem Einfluss neuer Technologien sind die klassischen Fächer marginalisiert worden, ohne dass den neuen genügend Platz für eine angemessene Entfaltung eingeräumt worden ist. Wem nützen wenige Lektionen Kommunikation, wenn gleichzeitig in der Physik Sprache durch Formelhüpfen ersetzt wird?

Modellbildung ist ein zentrales Element wissenschaftlicher Tätigkeit. Die Fähigkeit, Daten und Fakten zu kausalen Mustern zusammen zu fassen, ist urmenschlich und generiert andauernd neue Wissenschaften und leider auch Scheinwissen wie Astrologie, Homöopathie oder Radiästhesie.

Visionäre Studiengänge wie Datenanalyse und Prozessdesign oder Aviatik stehen weniger unter Druck von Tradition und vorgegebenem Berufsbild. Entsprechend bieten sie Raum für neue Fächerkombinationen und grosszügige Stundendotation. Die nachfolgende Darstellung basieren auf den Erfahrungen im Fach "Physik und Systemwissenschaft für Aviatik”. Dieses Modul, das von
Lehrkräften aus der Physik und dem Sprachbereich gemeinsam unterrichtet worden ist, hatte folgenden Aufbau

- Plenum: Flipped Classroom im Hörsaal

- Labor: Übungen und Modellbildung in Kleinklassen

- Kommunikation: Bericht, Vortrag, Poster, Reportage in Kleinklassen.

Ziel des vorliegenden Aufsatzes ist nicht ein Resümee zu diesem mittlerweile schon wieder fragmentierten Modul, sondern eine Vision zu Modellbildung und Simulation als integrierendes Element eines zeitgemässen Studienganges für Ingenieure, Naturwissenschaften und Medizinalberufe.

\section{Hydrodynamik}

Der herkömmliche Einstieg über die Punktmechanik in die Physik verhindert, dass bei den Studierenden kreative Prozesse aktiviert werden. Das Modell von Massenpunkten, die sich unter der Fernwirkung von Kräften in einem gegebenen Raum bewegen, war damals im Barock ein geniale Leistung, ist aber derart weit von unseren Erfahrungen entfernt, dass es von den Studierenden kaum verstanden wird [1]. Leider scheitert oft auch die Übertragung auf alltägliche Problemstellungen, wie das Beispiel der falsch eingezeichneten Kräfte bei der schiefen Ebene zeigt [2].

Dieser kaum zu behebende Mangel der Newtonschen Mechanik sowie der in dieser Theorie nur implizit vorhandene Energiebegriff haben uns bewogen, über die Hydrodynamik in die Naturwissenschaften einzusteigen. Mit dem Volumen oder der schweren Masse als bilanzierfähige Mengen sowie dem Druck bzw. dem Gravitationspotential als zugehörige Energiebeladung stehen Grössen im Zentrum, die jeder kennt oder von denen eine ausbaufähige Vorstellung vorhanden ist. Im Gegensatz zur Punktmechanik oder zur klassischen Thermostatik werden nicht nur Speicher, sondern gleichberechtigt auch Stromglieder untersucht. Die Hydrodynamik liefert zudem das Vorbild für das Flüssigkeitsbild [3], eine arche- 
typische Darstellung, die in vielen Zweigen der Naturwissenschaften eingesetzt werden kann.

Die Hydrodynamik ermöglicht einen intuitiven Einstieg ins systemdynamische Modellieren. Man kann darüber streiten, ob ein Räuber-Beute-Modell mit Schneehasen und Luchsen anschaulicher ist als ein Modell zu den drei Seen im Berner Seeland. Von der Physik über die Chemie bis zur Pharmakokinetik ist aber das Bilanzieren kontinuierlicher Mengen eine derart zentrale Tätigkeit, dass diese Fähigkeit nicht früh genug geübt werden kann. Im Studiengang Aviatik sind wir mit dem Ausflussgesetz von Torricelli eingestiegen [4], haben danach die Dynamik kommunizierender Gefässe studiert [5], um zuletzt in einer etwas ausführlichen Übung den Druckausgleich zwischen zwei PET-Flaschen zu untersuchen [6]. Dieser Aufbau erlaubt eine systematische Einführung in die grundlegenden Strukturen wie Volumen- und überlagerte Energiebilanz sowie das Studium von kapazitiven, resistiven und induktiven Gesetzen, wobei speziell in der Hydrodynamik viele Abhängigkeiten nichtlinear sind. In zwei der drei Beispiele haben wir das gespeicherte Wasservolumen indirekt über eine Kraftmessung erfasst, indem wir die Töpfe und Flaschen am Haken eines entsprechenden Messgerätes aufgehängt haben.

\section{Energieträger}

Neben den bilanzierbaren Mengen, den zugehörigen Potentialen sowie den konstitutiven Gesetzen bildet die Energie die Buchhaltungsgrösse. Über die Energie werden die verschiedenen Gebiete miteinander vernetzt, d.h. die Energie ist eine Art Währung der Physik. Tabelle 1 gibt einen Überblick über die Energieträger und die zugehörigen Potentiale.

Für alle Mengen kann eine Bilanzgleichung formuliert werden

$$
\sum_{i} I_{M_{i}}+\Sigma_{M}+\Pi_{M}=\dot{M}
$$

I steht für Stromstärke, $\Sigma$ für Quellenstärke, $\Pi$ für Produktionsrate und $M$ für eine der Mengen. Einige Mengen wie Masse oder Volumen werden nur zusammen mit der Materie transportiert, andere wie die Ladung, der Impuls oder die Entropie fliessen auch durch die Materie hindurch.

Der Energietransport ist in den meisten Fällen durch folgende Beziehung an die entsprechende Menge gebungen

$$
I_{W}=\varphi_{M} I_{M}
$$

$W$ steht für Energie und $\varphi$ für Potential. Formel (2) liefert beim Impuls- und beim Drehimpulstransport ein Skalarprodukt. Bei einigen konvektiven Transporten wie Impuls in bewegten Stoffen oder dem Elektronenstrahl kann Formel (2) nicht angewendet werden.

\begin{tabular}{lll}
\hline Menge & Potential & Gebiet \\
\hline Volumen $V$ & Druck & Hydraulik \\
\hline Masse $m$ & Gravitationspotential & Hydraulik \\
\hline Ladung $Q$ & elektrisches & Elektrodynamik \\
\hline Impuls $\boldsymbol{p}$ & Geschwindigkeit & Mechanik \\
\hline Drehimpuls $\boldsymbol{L}$ & Winkelgeschwindigkeit & Mechanik \\
\hline Entropie $S$ & Temperatur & Thermodynamik \\
\hline Stoffmenge $\boldsymbol{n}$ & chemisches & Chemie \\
\hline
\end{tabular}

Tabelle 1: Die Energieträger und ihre Potentiale. Impuls und Drehimpuls werden durch ein raumfestes Koordinatensystem in je drei Mengen unterteilt. Diese sechs Mengen sind wie die elektrische Ladung vorzeichenfähig. Volumen, Entropie und Stoffmenge sind keine Erhaltungsgrössen.

Fällt ein Mengenstrom über eine zugehörige Potentialdifferenz, wird Energie aufgenommen oder abgegeben. Die Prozessleistung $P$ folgt aus Formel (2)

$$
P=\left(\varphi_{M_{1}}-\varphi_{M_{2}}\right) I_{M}
$$

Gemäss Formel (3) ist die Leistung positive, wenn Energie freigesetzt wird, wenn der Mengenstrom $I_{M}$ vom hohen zum tiefen Potential fällt. Eine freigesetzte Leistung wird meist nur teilweise von einem zweiten Prozess aufgenommen. Der Rest dient der Entropieproduktion. Die zugehörige Produktionsrate ist gleich dissipierte Leistung geteilt durch die dort herrschende absolute Temperatur. Das hier verwendete Energieträgerbild des Karlsruher Physikkurses (KPK) [7] hat grosse Ähnlichkeit mit der Bond-Graphen-Theorie [8] oder dem Basiskonzept von Modelica [9].

\section{Kontinuumsmechanik und Relativitätstheorie}

Der KPK welcher der Systemphysik zugrunde liegt, ist vor Jahren durch eine Expertengruppe der Deutschen 
Physikalischen Gesellschaft massiv verunglimpft worden [10]. Dieses Ereignis, das wie ein Damoklesschwert auch über der Systemphysik schwebt, verlangt nach einer wissenschaftlichen Begründung der nachfolgenden Ausführungen. Die Mechanik hat seit Newton einen intensiven Ausbau erfahren und mit der Kontinuumsmechanik einen vorläufigen Abschluss gefunden. Der Hinweis, dass die hier dargelegte Translationsmechanik auf der Impulsbilanz im Sinne der Navier-Stokes-Gleichung beruht, sollte als Begründung genügen. Weil weder Claude Louis Marie Henri Navier noch George Gabriel Stokes im physikalischen Olymp nicht annähernd so hoch anzusiedeln sind wie Sir Isaac Newton, rufe ich Albert Einstein als Zeuge auf. Die wohl grösste Leistung Einsteins war die Verallgemeinerung der Newtonschen Gravitationstheorie. Wo bei Newton gemäss der Formulierung von Carl Friedrich Gauß die Massendichte steht, setzte Einstein den Energie-Impuls-Tensor. Diese Grösse beschreibt die Verteilung von Energie und Impuls in der Raum-Zeit, wobei die Gesamtenergie eines Körpers gleich seiner Masse mal die Lichtgeschwindigkeit im Quadrat ist. Schreibt man den Energie-Impuls-Tensor bezüglich eines ausgewählten Koordinatensystems, erhält man eine vier-mal-vier Matrix

$$
T^{\alpha \beta}=\left[\begin{array}{llll}
T^{00} & T^{01} & T^{02} & T^{03} \\
T^{10} & T^{11} & T^{12} & T^{13} \\
T^{20} & T^{21} & T^{22} & T^{23} \\
T^{30} & T^{31} & T^{32} & T^{33}
\end{array}\right]
$$

Nimmt man als nullte Koordinate die Lichtgeschwindigkeit mal die Zeit, steht in der ersten Zeile die Energiedichte sowie die Energiestromdichte geteilt durch die Lichtgeschwindigkeit. Die restlichen drei Zeilen beinhalten die Impulsdichte mal die Lichtgeschwindigkeit sowie die Impulsstromdichte.

Als einfachen Anwendungsfall setzen wir uns auf den Riemen eines Riementriebs und formulieren den Energie-Impuls-Tensor für einen materiellen Punkt. Indem wir die $x$-Achse längs des Riemens ausrichten, können wir den Tensor in nur zwei Dimensionen formulieren

$$
T^{\alpha \beta}=\left[\begin{array}{cc}
\rho c^{2} & 0 \\
0 & -\sigma
\end{array}\right]
$$

Die Energiedichte ist gleich Massendichte mal Lichtgeschwindigkeit im Quadrat. Weil die Zugspannung $\sigma$ als positive Grösse definiert wird, die zugehörige Impulskomponente bei dieser Belastung gegen die eigene Koordinatenrichtung fliesst, muss ein negatives Vorzeichen beigefügt werden.
Transormiert man den mit Formel (5) beschriebenen Energie-Impuls-Tensor ins Ruhesystem des Riementriebs, erhält man den folgenden Ausdruck

$$
\begin{gathered}
T^{\alpha \beta}=\gamma^{2}\left[\begin{array}{cc}
\rho c^{2}-\beta^{2} \sigma & \rho v c-\beta \sigma \\
\rho v c-\beta \sigma & \rho v^{2}-\sigma
\end{array}\right] \\
\beta=\frac{v}{c} \quad \gamma=\frac{1}{\sqrt{1-\beta^{2}}}
\end{gathered}
$$

Weil das Verhältnis $\beta$ von Riemengeschwindigkeit $v$ zur Lichtgeschwindigkeit $c$ etwa $10^{-7}$ beträgt, ist der Lorentzfaktor $\gamma$ sehr nahe bei eins. Nimmt man nur die relevanten Terme, erhält man folgende Näherung

$$
T^{\alpha \beta} \approx\left[\begin{array}{ll}
\rho c^{2} & \rho v c-\frac{v \sigma}{c} \\
\rho v c & \rho v^{2}-\sigma
\end{array}\right]
$$

Die Massendichte ändert sich nicht messbar, dafür speichert der Riemen Impuls mit der Dichte $\rho v$. Die zweite Kolonne liefert zwei bemerkenswerte Einsichten: neben der Massensromdichte $\rho v$ erscheint im oberen Element noch eine Energiestromdichte $-v \sigma$; im unteren Element taucht neben der leitungsartigen Impulsstromdichte - $\sigma$ noch eine konvektive Impulsstromdichte $\rho v^{2}$ auf.

Impuls kann leitungsartig durch und konvektiv mit der Materie transportiert werden. Bei Zugspannung fliesst der Impuls rückwärts und bei Druckspannung vorwärts. Konvektiv wird der Impuls in beiden Riemen vorwärts transportiert. Würde man die Geschwindigkeit des Riemens erhöhen, bis das vierte Element in Formel (7) gleich null ist, stünde der Riemen unter Zugspannung, würde aber über den Riemenscheiben abheben. Formel (7) zeigt uns, dass ein mechanischer Energietransport nur zusammen mit einem leitungsartigen Impulsstrom erfolgen kann, wobei die Geschwindigkeit den Zusammenhang herstellt. Dieser Transport kann mit einem punktmechanischen Modell nicht erklärt werden. Die kinetische Energie, die im Riemen gespeichert ist, findet man in Formel (7) erst, wenn man den Lorentzfaktor $\gamma$ nach $v$ entwickelt.

\section{Translationsmechanik}

Stellvertretend für das ganze Kapitel sei hier ein Praktikumsversuch beschrieben. Zwei Wagen auf einer Rollbahn sind über Gummifaden untereinander sowie mit den beiden Enden der Bahn verbunden. In Vorversuchen wird die Reibung der Wagen und die statische Kennlinie der Gummifaden bestimmt. In einem ersten Experiment wird 
ein Wagen, der mit zwei Gummifaden an der Bahn fixiert ist, in Schwingung versetzt. Gemessen werden der Ort mittels eines Distanzsensor (Ultraschall oder Laser), die Impulsstromstärken in beiden Gummifaden sowie die Beschleunigung des Wagens [11]. Parallel dazu wird ein systemdynamisches Modell erstellt. Die Parameter eines rheologischen Gummifaden-Modells werden so lange verändert, bis die Mess- und Simulationsdaten optimal übereinstimmen. Zur Validierung werden zwei Wagen mit mit einem Gummifaden untereinander sowie mit zwei weiteren mit der Bahn verbunden. Diese Anordnung wird wiederum in ein systemdynamisches Modell abgebildet. Diesmal dürfen die Parameter nicht mehr verändert werden. Ein Vergleich der Mess- mit den Simulationsdaten zeigt, wie präzis das Modell die Bewegung der beiden Wagen vorherzusagen vermag.

In einem Bericht müssen die Studierenden die $\mathrm{Zu}-$ sammenhänge im Impulsstrombild, im Flüssigkeitsbild und im Kraftbild erklären [12]. Eine ausführliche Beschreibung des systemdynamischen Modells und des experimentellen Aufbaus sowie eine gründliche Diskussion der Mess- und Simulationsresultate werden ebenfalls eingefordert. Bild 1 zeigt das Systemdiagramm (Flowchart) dieses Experiments, Bild 2 die überlagerte Energieebene.

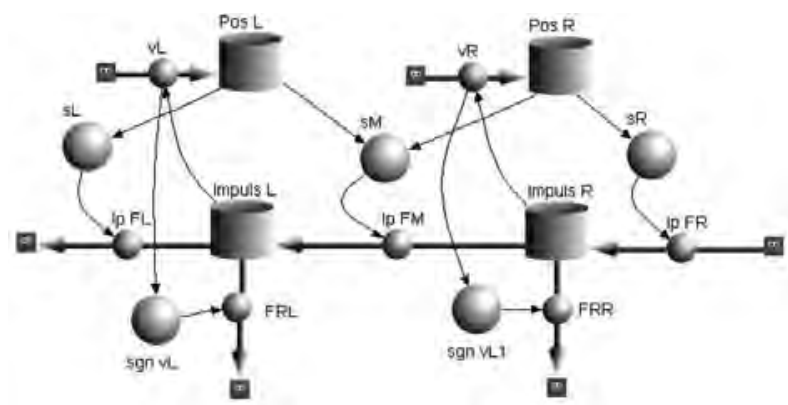

Bild 1: Systemdiagramm der zwei Wagen mit drei Gummifaden sowie den beiden reibungsbedingten Impulsströmen. Die oberen beiden Töpfe integrieren die Geschwindigkeit zum Ort.

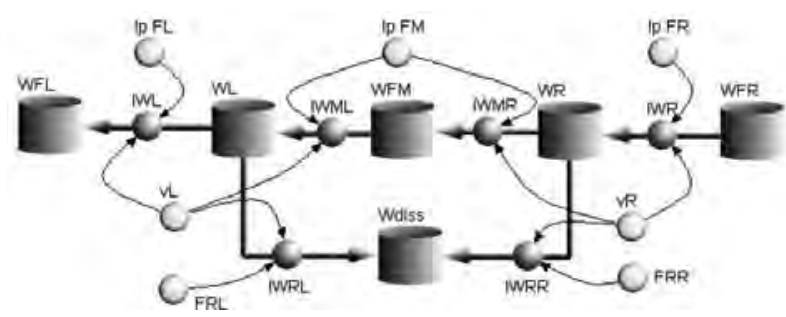

Bild 2: Die Energiebilanz rekapituliert die Impulsbilanz, wobei die Gummifaden und die Reibschicht zusätzlich Energie speichern können. Deshalb enthält die Energieebene mehr Töpfe als die Impulsebene.
Dieses Experiment lässt sich in vielen Varianten durchführen. Die Gummifaden können durch Stahlfedern oder durch starke Magnete ersetzt warden. Als Reibelement darf auch mal eine Wirbelstrombremse oder ein Segel eingesetzt werden. Anhand dieses Beispiels erkennt man deutlich den Unterschied zwischen der Punktmechanik und der Systemphysik, befasst sich erstere nur mit der Bewegung der Wagen, so verschiebt letztere den Fokus auf die Impuls- und Energieströme.

\section{Thermodynamik}

Wer die Wärme auf die Energie reduziert, hat gemäss dem ersten Hauptsatz der Thermodynamik recht, verletzt aber die dort getroffene Definition, sobald er die Wörter Wärmespeicher, Wärmedurchgang und Wärmeproduktion in den Mund nimmt. Wer dagegen Entropiespeicher, Entropietransport und Entropieproduktion sagt, trifft den Kern der Thermodynamik. Entropie steht für die thermische Basismenge. Entropie wird in der Wärmepumpe von tiefer zur hohen Temperatur gefördert. Entropie wird im Haus gespeichert und Entropie fliesst wieder an die Umgebung weg. Weil die Energie eine Erhaltungsgrösse ist, eignet sie sich für gewisse Fragestellungen wie Wärmedurchgang oder Wärmespeicher besser als die vermehrungswillige Entropie. Doch spätestens beim Optimieren von Sonnenkollektoren, Wärmepumpen oder auch Verbrennungsmotoren muss die Entropie wieder ins Spiel gebracht werden.

Die Thermodynamik beschäftigt sich oft mit der Schnittstelle zwischen Thermik und Mechanik. Um ein gewisses Grundverständnis dafür zu erwerben, modellieren die Studierenden in einem ersten Schritt den Carnotor [13]. Danach wenden sie sich einer speziellen Fragestellung zu, zum Beispiel dem Laborversuch von Rüchardt [14]. Bei der an der ZHAW verwendeten Version schwingt eine zweckentfremdete Milchpumpe über einem Erlenmeyer-Kolben. Das dynamische System besteht demnach aus einem vertikal beweglichen Körper und einer Gasfeder. Indem man den Erlenmeyer mit Stahlwolle, Aluminiumfolie oder Watte füllt, verändert sich der Wärmeleitwert. Das zugehörige systemdynamische Modell formuliert die Bilanz für Entropie und Volumen sowie für die beide Mengen verbindende Energie [15].

Bild 3 zeigt zwei Simulationsresultate im Temperatur-Entropie-Diagramm. Die Extremfälle wären die isentrope (vertikale Linie) und die isotherme Schwingung 
(horizontale Linie). Schaltet man die mechanische Reibung im Modell aus, tritt in diesen beiden Grenzprozessen keine Dämpfung auf. Das Dämpfungsmaximum liegt irgendwo dazwischen und kann mittels einer Parameterstudie schnell gefunden werden. Zudem verändert sich die Schwingungsdauer zwischen den beiden Extremfällen um etwa $20 \%$. Beide Phänomene lassen sich auch experimentell nachweisen, indem die Füllmenge im Erlenmeyer variiert wird. Der Erfolg dieser Lernmethode ist augenfällig. So haben die Studierenden bei der Präsentation ihrer Arbeit in der Regel ein erstaunlich gutes Verständnis für die Thermodynamik gezeigt.

\section{Temperatur-Entropie-Diagramm: T(S)}

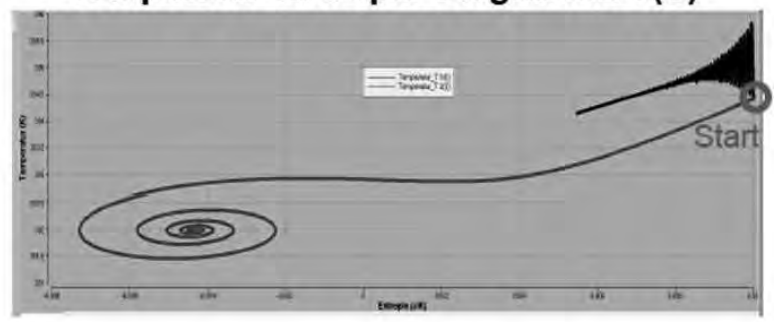

Bild 3: Das T-S-Diagramm für kleinen (schwarz) und grossen (roten) Leitwert.

\section{Modelica}

Studierende setzen heute die unterschiedlichsten Simulationstools ein, welche für spezielle Fragestellungen entwickelt worden sind. Dazu müssen sie weder die Theorie noch die Mathematik verstanden haben. Steht für ein gewisses Problem kein geeignetes Werkzeug zur Verfügung, wird oft Simulink benutzt. Simulink ist kein Modellierunstool im engeren Sinn, was sich in der Anwendung zeigt. So werden die Differentialgleichungen meist händisch aufgestellt und nur zur Lösung in Simulink eingegeben. Systemdynamische Tools wie STELLA, Berkeley Madonna und Vensim eignen sich besser als Simulink, um dynamische Systeme zu analysieren und abzubilden, wie auch Andwendungsbeispiele aus der Ökonomie und der Ökologie zeigen. Man kommt mit der graphischen Eingabe und der Numerik aber schnell an Grenzen, wenn die Systeme komplexer werden. Zudem ist die Wiederverwendbarkeit einzelner Teilmodelle nur beschränkt möglich. Modelica kann die Lücke zwischen der hier dargelegten, auf Verständnis und Einsicht beruhenden Basisausbildung und den Ansprüchen vieler Anwender schliessen.

Komplexe Systeme können auch in Modelica ohne tiefes Verständnis der Theorie abgebildet werden, indem man die einzelnen Komponenten nach dem Lego-Prinzip der Bibliothek entnimmt, graphisch zusammengefügt und entsprechend den Vorgaben parametrisiert. Wenn das nicht funktioniert, liegt es meist am fehlenden Verständis des Anwenders, manchmal aber auch an den in den Komponenten hinterlegten Gleichungen.

Zu Modelica gehört eine Standardbibliothek, die von der Hydraulik über die Mechanik und die Elektrik bis zur Thermodynamik reicht. Statt diese Bibliothek zu benutzen und zu ergänzen, plädiere ich für eine spezielle Bibliothek, welche die zentralen Ideen der Systemphysik rekapituliert [16]. Obwohl schon die Standardbibliothek methodisch der Systemphysik sehr nahesteht, können ein paar Dinge besser gemacht werden. Dazu gehört die Definition der Konnektoren, die konsequente Trennung von Speicher und Stromglieder sowie der konsequente Umgang mit der Energie. Dazu zwei Beispiele und eine allgemeine Anregung.

Die Unterbibliothek Translation enthält die Masse als Speicher sowie Feder und Dämpfer als Stromglieder. Daneben findet man aber auch eine Masse mit Reibung. Konsequent wäre ein Modell für die Masse und verschiedene Modell für die Impulsleiter, womit auch die unterschiedlichsten Reibmodelle gemeint sind. Zudem sollte die Masse auch eine Impulsquelle für die Gravitationskraft aufweisen, was ganz im Sinne von Albert Einstein wäre.

Die Konnektoren für die Thermodynamik weisen als «flow»-Grösse den Energiestrom aus. Hier sollte wie in allen anderen Domänen die Stromstärke der Basismenge, also der Entropie, eingesetzt werden. Was auf den ersten Blick nach l'art pour l'art aussieht, liefert auf den zweiten eine wesentliche Verbesserung. Solange man maximal dissipative Systeme wie Motorenkühlung oder Gebäudeisolation modelliert, reicht eine auf dem Energiebegriff aufgebaute Bibliothek völlig aus. Nicht aber, wenn man thermodynamische System wie Wärmepumpen oder das weiter oben erwähnte Rückardt-Experiment modellieren will. Die Energieerhaltung für die Wärmeleitung lässt sich auch formulieren, wenn man die Entropie als Menge und die absolute Temperatur als Potential nimmt.

Die Konnektoren für die verschiedenen Gebiete sollten wenn möglich entsprechend Tabelle 1 ausgeführt werden. Zudem sollte man die Energie überall mitmodellieren, damit die Energiebetrachtung immer dann zur Verfügung steht, wenn sie gebraucht wird. Bei der Wärmeleitung und in allen andern dissipativen Elementen sollte zusätzlich die Entropieproduktion gerechnet wer- 
den. Aus didaktischer Sicht sind diese Anpassungen notwendig, um die Erkenntisse aus der Grundausbildung zu repetieren und zu vertiefen. Welchen Gewinn man mit der konsequenten Umsetzung der systemphysikalischen Ideen in Bezug auf Variationsbreite und Stabiltität erhält, kann nicht zum Voraus gesagt werden. Die Erfahrungen beim Aufbau der Anwenderbibliothek DyMoRail haben mir gezeigt, dass man sich viel Ärger ersparen kann, wenn man sich konsequent an die Struktur der Systemphysik hält [17].

\section{Referenzen}

[1] Hestenes D, Wells M, Swackhamer D. Force concept inventory. The Physics Teacher. 1992; 30. 141 - 158. doi 10.1119/1.2343497.

[2] Meschede, D. Gerthsen Physik. 24. Auflage. Berlin Heidelberg: Springer; 2010. Seite 78.

[3] Maurer, W: Der Impuls im Flüssigkeitsbild. Praxis der Naturwissenschaften - Physik für die Schule. 1996. 4/45: 12-16.

[4] Maurer, W. Ausflussgesetz von Torricelli. Youtube 24.09.2015: https://youtu.be/-giLthDilGY

[5] Maurer, W. Dynamik kommunizierender Gefässe. Youtube 23.09.2014: https://youtu.be/xNki45iOe3c

[6] Maurer, W. Druckausgleich modellieren. Youtube 03.05.2019: https://youtu.be/NT0adWQMMoo

[7] Herrmann, F. Der Karlsruher Physikkurs. http://www.physikdidaktik.uni-karlsruhe.de/

[8] Karnopp D, Margolis D, Rosenberg R. System Dynamics - A Unified Approach. 2ed. New York: Wiley; 1990.

[9] The Modelica Association. https://www.modelica.org/

[10] Lehn R. und andere. Gutachten über den Karlsruher Physikkurs. In Auftrag gegeben von der Deutschen Physikalischen Gesellschaft. 28. Februar 2013.

https://www.dpg-physik.de/veroeffentlichungen/publikationen/stellungnahmen-der-dpg/bildung-wissenschaftlicher-nachwuchs/kpk/stellungnahme_kpk.pdf

[11] Maurer, W. Modellbildung: zwei Wagen auf der Rollbahn. Youtube 05.12.2016: https://youtu.be/5a31p3uWBXQ

[12] Maurer, W. Rangierstoss. Youtube 23.05.2019: https://youtu.be/2LH3LHhMQg0

[13] Maurer, W. Der Carnotor. Youtube 19.06.2011: https://youtu.be/1QAQJV2C6B0

[14] Wikipedia. Rüchardt-Experiment. https://de.wikipedia.org/wiki/R\%C3\%BCchardt-Experiment

[15] Maurer, W. Rüchardt-Experiment. Youtube 08.04.2014: https://youtu.be/8Tcw_yKcpsM

[16] Maurer, W. PhyDynSys - eine Modelica-Bibliothek zur Physik der dynamischen Systeme. ASIM 19. Symposium Simulationstechnik. Hannover 12.-14. September 2006.
https://www.academia.edu/32931337/PhyDynSys_eine_Modelica-Bibliothek_zur_Physik_der_dynamischen_Systeme

[17] Maurer, W. Simulationsgestützte Entwicklung von Puffern und Dämpfern für Eisenbahnzüge. ASIM 18. Symposium Simulationstechnik. Erlangen 12.-15. September 2006. 